\title{
Exploration of the Resources Cooperating Profit Relationship at the Internet Age
}

\author{
Han Zhang ${ }^{1, \text { a }}$ \\ ${ }^{1}$ Kunming University of Science and Technology-Oxbridge College, Department of Economics and \\ Management, Kunming, Yunnan, China, 650000 \\ aemail,
}

Keywords: Resources Cooperating, Profit Relationship, Internet Age, Exploration

\begin{abstract}
With the development of the Internet, the gradual rise of mass collaboration and showing more and more widely momentum, mass collaboration to subvert the traditional concept of resource ownership, use the rules to ensure that everyone involved can share resources, collaborate to jointly to an end, and the distribution of resources throughout the body linked to the exchange of ideas and information, exchange of resources, the formation of a large-scale collaboration of resources on the Internet network.
\end{abstract}

\section{Introduction}

Internet mass collaboration network of resources to break the pyramid-like hierarchical structure, hierarchical structure change any members of the network structure, network structure can freely communicate with each other to share ideas, information and resources, but also broke the limits of time and space distribution of resources around the world body can communicate and share resources from anywhere. Resource economy falls, the rise of resource management, making the sharing of resources in the resource body status and increasingly prominent role. Share resources between different areas or disciplines, has become an important issue.

With the rapid development of Web2.0 applications, the Internet is increasingly reflected by the organization, openness, decentralization, polymeric, highly interactive and innovative characteristics, the grassroots on the Internet is playing an increasingly important role. Due to profound changes in technology, population, business, economics and the world, we are entering an era of unprecedented public participation in economic activities. This large-scale collaboration and participation of the public on the Internet has also become a new model, produced an unexpected impact, is playing an increasingly important role. Mass collaboration (Mass Collaboration) concept was first "wiki economics," a book proposed by Tapscott and Williams (2006), they think the new mass collaboration is changing the corporate and social resources and the ability to innovate and create value way, and it pointed out that the future economic development will follow a large-scale collaborative way to organize. The book presents four new laws of this model: open, peering, sharing, and global operations. At the same time with plenty of examples of organizations and individuals how to use the new rules to promote innovation in the workplace, the community and industry. But the author did not give adequate definitions and criteria to define a large-scale collaboration. MarkAlan Elliott described the mass collaboration for distributed, collaborative participants often did not appear linked to the structure of cooperation. He also believes that large-scale cooperation is the most developed and extended collective human creative process currently available and carry out work stimulate collaboration intersection. This paper argues that, simply put, is a large-scale collaboration on the Internet, together with the large number of participant involved in particular joint collaborative activities plays a collective effect. After Tapscott and Williams proposed the concept of large-scale collaboration, the mass collaboration is the Internet as a new pattern of behavior has been widely applied to the sciences, business, education, software development, medical research and other fields. 


\section{The Relevant Theories Basis}

Network Effects. Network effect theory is an important economic thinking with the ICT revolution generated. Information there is an inherent need for interconnection, because people produce and use their purpose is to improve the collection and exchange of information. Meet this demand level is closely related to the size of the network. Only a user's network is worthless. If the network has only a few users, they not only have to bear the high operating costs and only the exchange of information and experience with a limited number of people. As the number of users increases, this situation is not conducive to economies of scale will continue to improve, the cost borne by each user will continue to decline, while the scope of the exchange of information and experience was expanded, all users from possible expansion of the network of get greater value. The case that the value of a product for a user depends on the use of the product which he used the number of households in economics said the network externalities, also known as network effects. Network effects can be divided into two "direct network effects" and "indirect network effects." That said former interconnected by the network effects caused by demand, and the latter with a complementary product-related. It is large-scale collaborative network effects of the Internet network resources reflect resource innovators and others if not connected, not open to innovations, his innovative resources will not be recognized and, therefore, lose the resources produce and Innovation meaning. If only a few people involved in collaboration, then the resource sharing network resources spread range will be very limited, resource collision will be reduced, the probability of generating innovation will be reduced. The massive participation and collaboration, share resources will accelerate the spread of collision with each other, providing opportunities for innovation resources.

Social Exchange Theory. Social exchange theory is a sociological theory of the Second World War in Western society circles gradually flourishing popularity of the theory of "norm of reciprocity" basis, that the reason why the exchange occurs because individuals expect social reward and this reward both social substances also it includes emotional. According to social exchange theory, if the participants are able to benefit from the participation of collaboration, but also comply with norms of reciprocity, are willing to provide in return for their income, then the composition of participants and other participants of the interactive community, interaction and influence, shared specific goals and expectations, social exchange process is really started. As a new social network, development of the Internet to provide participants with a new communication environment and space, although the relationship between the members of the network within the community relatively vague, sense of community in terms of consistency, specifications, objectives and action groups are not as realistic but the Internet breaks through the limitations of time and space, users can collaborate arbitrarily join or leave a network or community groups; while participants in the size and degree of interaction can significantly stretch, in the field of communication unlimited fine min or integration.

Immersion Theory. Immersion theory is put forward by Csikszentimihalyi in the late seventies the theory and immersed as "actors when certain activities, awareness concentrated in a very narrow range of unrelated ideas and perceptions are filtered out, only to have specific goals and clear feedback response." According viewpoint immersion theory, it is based on intrinsic motivation tends to do what he wanted, and immersive experience is inherent in the individual prototype driven by motives state of consciousness, is that people are willing to constantly engage in certain specific activities of the underlying reasons . Early immersion theory is mainly used for living, working, recreation, sports, reading and other activities, and the current due to the rapid development of the Internet, immersed theory is introduced into the study of the interactions of the network. Hoffman and Novak to Csikszentimihalyi of research-based, using the "immersion" variables to define and measure network environment level experience, they studied for the customer network browsing behavior indicates that flow state has the following characteristics: Reaction computer interaction speed fluency; pleasantness; lost of self-consciousness; the results of the self-reinforcing. To generate immersive experience in the activity, the user must strike a balance between challenge and skill involved in activities they have in. Participation initiative of this research collaboration, in 
addition to the initiative refers to the main body interaction and initiative, the initiative also refers to the body's own platform and virtual interaction. When the self-interaction and virtual environments, interactive initiative body feels fast, and there is a sense of pleasure, so that self-consciousness temporarily disappear into the flow state, it is possible to obtain a strong perception of behavioral control, stronger exploratory behavior and participation, Therefore, the main initiative willing to participate in online collaboration.

\section{The Main Aspects of the Resources Collaboration in Internet Era}

The Dynamic Spectrum Collaboration in the Cognitive Cellular Networks. For the cellular network, the study of dynamic current sharing wireless communications is the most popular cognitive cellular network available licensed spectrums. Through the establishment of the existing cellular network with dynamic spectrum sharing model with cognitive function secondary access network efficiently solve the problem of low utilization licensed spectrum, can ease the strain on the resources of the reality of the spectrum constraint to a certain extent. For cognitive cellular network characteristics, this paper based on external perception proxy dynamic spectrum access models. In this model, the establishment of a dynamic collaboration model to match the available spectrum resources based on user needs a dynamic matching between theory, to achieve a dynamic spectrum access networks available in licensed spectrum resource efficient management and utilization. In order to accelerate the practical application scenarios of the spectrum resource access procedure, a distributed fast spectrum sharing algorithms.

The Resources Dynamic Collaboration in the Self-Organizing Automotive Business Distribution. Regardless of the current rapid popularity of wireless LAN technology, distribution network and access transmission rate of the economy and other factors, based on self-organizing wireless LAN technology vehicle user networking method is currently the most popular wireless communication network in the field of research direction. To overcome the limited wireless local network coverage limitations and restrictions of movement, aiming at self-organizing vehicle access and wireless LAN business distribution applications, the research vehicle based on the current user how traffic flow to collaborate and share business needs its own communications resources , and ultimately vehicle business quickly and efficiently distribute applications. Proposed and established a complete set of business collaboration download and distribution methods; proposes a novel vehicle for highway traffic distribution chain cluster topology model to car sharing in the cluster user computing, communications and storage resources; raised collaboration for a highway scene clustering download performance evaluation model and content forwarding time. Business collaboration download and distribution methods will be studied highway vehicle applications flexible distribution network providing content ideas.

Wireless LAN Medium Access Control Optimization for Car Users. Car users to overcome as wireless LANs limited coverage limitations and restrictions of movement, dynamic collaboration is proposed based on self-organizing wireless LAN onboard service distribution methods and resources. To further improve vehicle dynamic user access to the wireless LAN performance itself, the paper also proposes a wireless local area network for universal access to in-car wireless LAN performance analysis and evaluation framework and the corresponding access control optimization. In this paper, based on the optimization of media access period access control method can be applied directly to the existing IEEE 802.11a / b / g network, not only do not need to modify the agreement, to better optimize network performance without compromising vehicle access fairness of media time and space to share. High-performance and flexible access to the proposed in-car wireless LAN access shared media optimization method for self-organized car users to provide guidance on the method.

\section{The Research on the Resource Collaboration Profit Relationship}

According to analysis, the scale of the global enterprise collaboration and open innovation in the coming years maintain more than $20 \%$ growth rate. But domestic cross-enterprise service is still in 
the initial stages of development, the country's small and medium micro enterprises 90 percent have not yet opened any cloud service implementation process, which is where the market gap. With the further transformation of small micro-enterprise philosophy of improvement, outsourcing non-core business will become a major trend in the domestic and international market prospects are very broad service.

Business Innovation and Collaboration Posted Resource Sharing is an enterprise itself does not have the lack of available resources, or because of factors such as cost control, innovation and hope to publish their own needs as a demand out there to the technology, resources and the ability of other enterprise cooperation to complete.

Innovation collaboration and service network-based information technology to complete its service jobs (including business and business processes) through the computer operation is complete, and the use of modern means of communication to deliver to enable enterprises by restructuring the value chain, optimizing the allocation of resources to reduce the costs and enhance the core competitiveness of enterprises.

Resources Cooperating Internet age has a number of innovative technologies, intelligent fast remote data transmission spoilers massive, cross-enterprise collaboration, real-time tracking and monitoring, visualization, transparent services and other characteristics, can provide visual workflow technology to strengthen computer technology in the cooperation process for the accurate and efficient resource management and deployment. In addition, the system also enables contractors to work flow and quality of the entire process of tracking and real-time monitoring, and can perform real-time settlement. The system not only to domestic small and medium micro enterprises provide a platform for online collaboration, but also provides the functionality of a technical trading results, and promote the transfer of paid domestic and international cooperation and technology to improve society as a whole resource efficiency.

\section{Conclusion}

Due to the profound changes in technology, population, business, economics and the world, we are entering an era of unprecedented public participation in economic activities. With the rapid development of Web2.0 applications, the Internet is increasingly reflected by the organization, openness, decentralization, polymeric, highly interactive and innovative characteristics, the grassroots on the Internet is playing an increasingly important role. These contributed to the formation of a collaborative network resources on the Internet, is the age of the Internet resource collaboration an important resource activities to help the parties to maximize profits.

\section{References}

[1] Chen Xiangdong, Li Jingyuan. China Educational Technology, Vol. 6 (2014) No 53, p.25-26

[2] Li Li, Wang Qunyong. Scientific Research, Vol. 12 (2015) No 27, p.74-76

[3] Jing Jianfen, Hou XuSiem. Information Science, Vol. 30 (2014) No 19, p.144-145

[4] Li Yong, Sun Jianhua. Industrial Technology Economy, Vol. 29 (2010) No 27, p.21-23

[5] Sun Jing. Science and Technology Management, Vol. 8 (2013) No 27, p.57-60 